\title{
Optimizing Reflector Selection for Indexing of EBSD Patterns via Dynamic Pattern Simulation
}

\author{
Stuart I. Wright ${ }^{1 *}$, Marc De Graef ${ }^{2}$, Saransh Singh $^{3}$ \\ 1. EDAX, St George UT, USA \\ 2. Department of Materials Science and Engineering, Carnegie Mellon University, Pittsburgh PA, USA \\ 3. Lawrence Livermore National Laboratory, Livermore CA, USA \\ * Corresponding author: stuart.wright@ametek.com
}

One challenge associated with indexing Electron Backscatter Diffraction (EBSD) patterns using conventional Hough-based indexing is selecting the appropriate set of reflectors. In early EBSD work, reflectors were sometimes chosen based on intensities listed in X-Ray powder diffraction tables. When the positions of the atoms in the crystal lattice are known, it is possible to calculate structure factors $\left(F_{h k l}\right)$ using the kinematic simulation of diffraction. In either case, reflectors with the highest-ranking structure factors are selected as a base set. However, this base set generally requires some iterative manual tuning against experimental patterns to attain an optimal set of reflectors. This is often a difficult process even for a skilled operator, particularly for lower symmetry materials. The better the base set, the easier the refinement procedure. Generally, the kinematic structure factors provide a better starting point for the manual tuning over X-Ray intensities. A new method [1] for ranking the reflectors based on integrating dynamical simulated electron backscatter intensities within a reflection (or band) over the full diffraction sphere [2] has been proposed. The ranking metric based on this integration for a given reflector $(h k l)$ we denote $\beta_{h k l}$. For simple structures such as face-centered-cubic structures, the dynamical $\beta_{h k l}$ based ranking closely follows the kinematical $F_{h k l}$ ranking. In more complex structures, the $\beta_{h k l}$ ranking can depart significantly from the $F_{h k l}$ ranking. In the materials investigated so far, the ranking based on the $\beta_{h k l}$ parameter tends to correlate better with the bands detected by the Hough transform than the $F_{h k l}$ ranking.

For a specific example case study, we have investigated the correlation between the dynamic model based $\beta_{h k l}$ ranking and the Hough transform band detection results for Rutile (tetragonal, $\mathrm{TiO}_{2}$ ) using both dynamically simulated EBSD patterns and experimental EBSD patterns. Various metrics based on the Hough transform have been investigated including the peak heights and frequency of detection by the Hough. For this work, we have used the Hough transform as implemented in EDAX's OIM Analysis $^{\mathrm{TM}}$ (a modified version of the released version 8.0). The default Hough detection settings in the software have been used. As the peak heights are not typically used in the indexing algorithm in EDAX's software [3], we focused simply on the average frequency a given reflector was detected by the Hough. Approximately 120,000 experimental patterns were collected on a polished Rutile sample. The scan area contained 1,125 individual grains. Dynamically simulated patterns were generated for 2,272 orientations based on cubochoric sampling of orientation space [4]. The spacing between orientations was approximately $10^{\circ}$. Figure 1 shows an orientation map and example of an experimental pattern and a simulated pattern. Table I compares the dynamical $\beta_{h k l}, F_{h k l}$ and Hough detection frequency $\left(f_{h k l}\right)$ rankings. We have also noted which reflectors were selected in the original manually refined structure file for indexing rutile. Note that the dynamic ranking suggests that some refinements to the list could improve the indexing of this phase. Some of the differences could potentially be mitigated by tuning of the Hough transform settings but some are due to subtle differences between the simulated and experimental patterns, in particular, the intensity profiles across the bands. 


\section{References:}

[1] SI Wright, S Singh, M De Graef, Microsc. Microanal., (submitted, 2019).

[2] AP Day, J. of Microscopy 230 (2007), p. 472.

[3] SI Wright, BL Adams, Metall. Mater. Trans. A 23 (1992), p. 759

[4] S Singh, M De Graef, Model. Simul. Mater. Sc. 24 (2016), p. 085013.

[4] We acknowledge GS Rohrer of CMU for supplying the Rutile sample and R Witt of EBSD Analytical for collecting the EBSD data. MDG and SS acknowledge an ONR Vannevar Bush Faculty Fellowship (N00014-16-1-2821), and the computational resources of the Materials Characterization Facility at CMU, grant MCF-677785. The work of SS was performed partially under the auspices of the U.S. Department of Energy at Lawrence Livermore National Laboratory under Contract DE-AC5207NA27344 (LLNL-JRNL-XXXXXX)
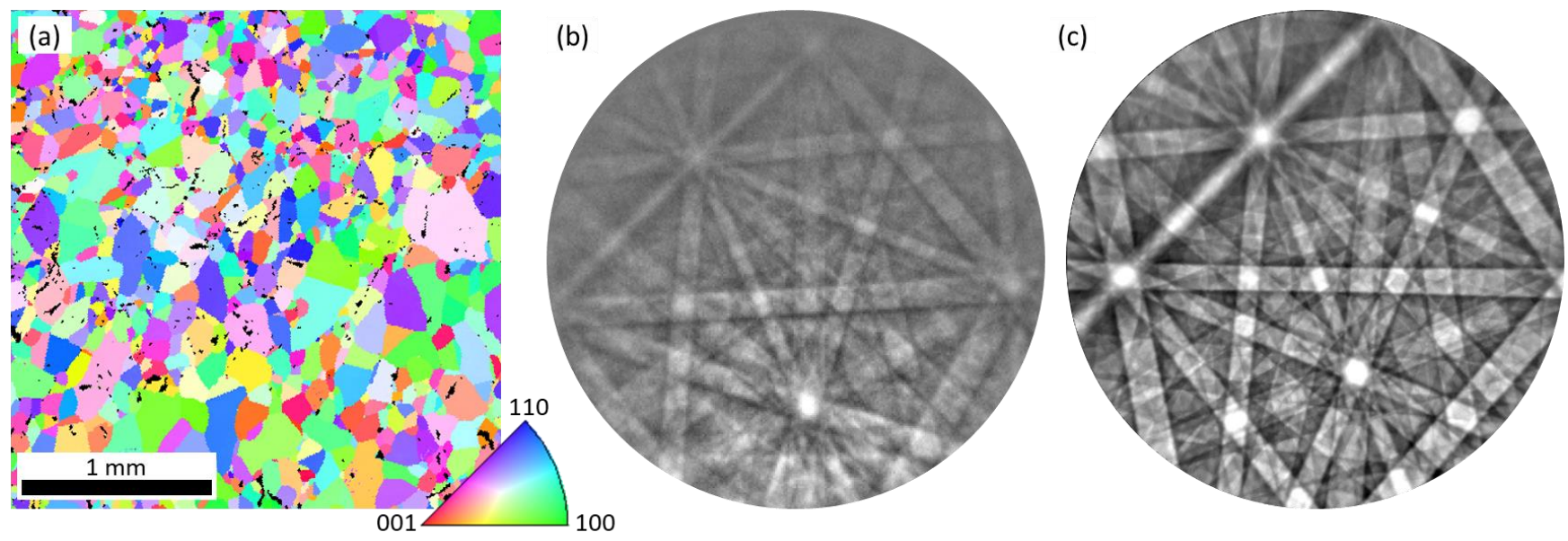

Figure 1. (a) Orientation map showing crystal direction aligned with the sample normal direction. (b) Example pattern selected from the map data shown in (a). (c) Dynamically simulated pattern for Rutile in a similar orientation and detector geometry as (b).

\begin{tabular}{cccccc}
\hline$h k l$ & $\beta_{h k l}$ & $F_{h k l}$ & $f_{h k l} E$ & $f_{h k l} S$ & $\begin{array}{c}\text { manual } \\
\text { selection }\end{array}$ \\
\hline 110 & 100.0 & 100.0 & 0.6 & 0.76 & - \\
002 & 76.2 & 45.0 & 0.4 & 0.45 & $\mathrm{x}$ \\
301 & 63.0 & 40.8 & 0.9 & 0.94 & $\mathrm{x}$ \\
211 & 57.7 & 52.3 & 1.0 & 0.99 & $\mathrm{x}$ \\
101 & 52.3 & 78.1 & 0.8 & 0.83 & $\mathrm{x}$ \\
111 & 46.3 & 0.0 & 0.6 & 0.89 & $\mathrm{x}$ \\
112 & 39.2 & 40.3 & 0.6 & 0.76 & $\mathrm{x}$ \\
400 & 31.9 & 32.9 & 0.3 & 0.73 & $\mathrm{x}$ \\
210 & 29.8 & 0.0 & 0.1 & 0.25 & $\mathrm{x}$ \\
332 & 28.2 & 22.1 & 0.0 & 0.07 & $\mathrm{x}$ \\
\hline
\end{tabular}

\begin{tabular}{cccccc}
\hline$h k l$ & $\beta_{h k l}$ & $F_{h k l}$ & $f_{h k l} E$ & $f_{h k l} S$ & $\begin{array}{c}\text { manual } \\
\text { selection }\end{array}$ \\
\hline 212 & 26.1 & 0.0 & 0.3 & 0.04 & - \\
310 & 25.5 & 44.1 & 0.4 & 0.87 & $\mathrm{x}$ \\
521 & 24.8 & 20.0 & 0.1 & 0.62 & - \\
510 & 23.3 & 23.2 & 0.1 & 0.02 & - \\
411 & 22.2 & 28.7 & 0.1 & 0.44 & $\mathrm{x}$ \\
213 & 21.9 & 22.7 & 0.1 & 0.07 & - \\
402 & 21.3 & 23.4 & 0.0 & 0.04 & - \\
312 & 17.3 & 28.5 & 0.1 & 0.11 & - \\
221 & 13.9 & 0.0 & 0.0 & 0.09 & - \\
720 & 3.5 & 0.0 & 0.1 & 0.06 & - \\
\hline
\end{tabular}

Table I. Dynamical $\left(\beta_{h k l}\right)$ and kinematical $\left(F_{h k l}\right)$ ranking factors and frequency of corresponding band detection in Hough transform from both experimental $\left(f_{h k l} E\right)$ and simulated $\left(f_{h k l} S\right)$ patterns for the twenty most prominent (according to $\beta_{h k l}$ ) reflectors in rutile. 